\title{
ELABORACIÓN DE UN CONTROL DE SERVOMECANISMO PARA LA CARACTERIZACIÓN ZONAL DE MATERIALES SEMICONDUCTORES POR LA TÉCNICA DE FOTORREFLECTANCIA
}

\author{
MAKING A SERVOMECHANISM CONTROL FOR THE SPATIAL VARIATION \\ CHARACTERIZATION OF SEMICONDUCTORS MATERIALS APPLYING \\ PHOTOREFLECTANCE TECHNIQUE
}

J. A. Acevedo-Londoño ${ }^{1}$, J. J. Prías-Barragán ${ }^{1,2}$, H. Ariza-Calderón ${ }^{1}$

'. Laboratorio de Optoelectrónica, Universidad del Quindío, A. A.2639 Armenia-Colombia.

2. Programa de Tecnología en Instrumentación Electrónica, Universidad del Quindío, Armenia-Colombia

Recibido: 22 de Abril del 2014

Aceptado: 29 de Septiembre del 2014

*Correspondencia: Laboratorio de optoelectrónica de la universidad del Quindío, Instituto interdisciplinario de las ciencias. Universidad del

Quindío, Carrera 15 calle 12 Norte, Armenia Quindío. Correo electrónico: jairo.acevedo@correounivalle.edu.co, jjprias@uniquindio.edu.co

\section{RESUMEN}

En este trabajo se presenta la automatización de un control electrónico digital para la caracterización zonal de materiales semiconductores, por medio de la técnica de Fotorreflectancia (FR) a temperatura ambiente. En este tipo de caracterización, la interacción de la radiación con la materia se logra dirigiendo dos haces de luz hacia la muestra mediante arreglos de espejos y lentes, lo cual requiere de una precisa alineación óptica. Por esta razón se agregó al camino óptico un servomecanismo para el control digital del posicionamiento de la muestra, dejando fija la zona de incidencia de los haces de luz, lo cual permitió desplazar en un sistema coordenado XY la muestra bajo estudio, lográndose resoluciones hasta de $1.0 \mu \mathrm{m}$ en ambos ejes. El control digital de la posición de estos haces de luz en la muestra, se logró mediante un sistema electrónico embebido basado en un microcontrolador AVR, ATMega 16 y una herramienta de visualización elaborada en el software LabVIEW de la empresa National Instruments. En la calibración del sistema de medida se emplearon monocristales comerciales de GaAs, lográndose reproducir información física reportada en la literatura especializada para este material.

Palabras claves: Fotorreflectancia, caracterización zonal, GaAs, automatización, servomecanismo

\begin{abstract}
This study is about the electronic automation of the photoreflectance technique (PR), which consisted of the development of a digital electronic control for the spatial variation characterization of semiconductor materials. In this type of optical characterization, the interaction of radiation with the matter is achieved by directing two beams of light toward the sample, using arrays of mirrors and lenses, which require precise optical alignment. For this reason, a servomechanism for digital control of the positioning of the sample was added to the optical path, fixing the incident area of the light beams, allowing moving the sample in the XY coordinate system under study, obtaining $1.0 \mu \mathrm{m}$ of resolution for both axes. The digital control of the position of these beams of light in the sample was achieved by embedding an electronic system based in AVR microcontroller, and a graphical user interface developed in LabVIEW software from the National Instruments. In the calibration of the measuring system, we used GaAs commercial single crystals, which are able to reproduce physical information reported in the literature for this material and therefore be able to contribute with this study to increasing the versatility of the PR technique.
\end{abstract}

Keywords: Photoreflectance, Mapping Characterization, GaA, automation, servomechanism 


\section{INTRODUCCIÓN}

El estado actual de la Ingeniería Electrónica se debe en parte al desarrollo de dispositivos de estado sólido y de nuevos materiales basados en tecnologías como la del silicio ( $\mathrm{Si}$ ) y la del Arseniuro de Galio (GaAs). El desarrollo de nuevos dispositivos a partir de materiales semiconductores ha sido posible gracias a la amplia e intensa labor de investigación científica sobre el tema. Los materiales semiconductores tienen un amplio rango de aplicabilidad que va desde detectores de rayos $\mathrm{X}$, circuitos integrados a frecuencias de microondas, hasta computadores, incluyendo dispositivos tan novedosos como los láseres semiconductores con emisión en diferentes longitudes de onda.

Entre las técnicas de caracterización no invasivas para el estudio de nuevos materiales semiconductores, se encuentran las que permiten obtener información acerca de sus propiedades ópticas, mediante la interacción de la radiación con el material a estudiar. Una de estas técnicas es la Fotorreflectancia (FR) que permite determinar con una alta resolución la energía de los puntos críticos de la estructura electrónica de bandas, así como la realización de estudios zonales en las muestras bajo estudio. Su aplicación incluye el estudio tanto de materiales en bloque como películas delgadas, multicapas y nano estructuras $(1,2)$.

Para la caracterización zonal de materiales semiconductores de una manera automatizada en este trabajo se logró construir un control de servomecanismo, que permitió el control digital del posicionamiento de un porta muestras, dejando fija la zona de incidencia de los haces de luz; es decir, se obtuvo un desplazamiento de la muestra en un sistema coordenado XY. Así mismo, mediante el desarrollo de este trabajo experimental realizado en el Laboratorio de Optoelectrónica del Instituto Interdisciplinario de las Ciencias de la Universidad del Quindío, se logró contribuir al incremento de la versatilidad de la técnica de Fotorreflectancia, por medio de la caracterización zonal de materiales semiconductores. Además, la calibración del sistema electrónico propuesto se realizó empleando monocristales comerciales de GaAs, lográndose reproducir información física reportada en la literatura especializada para este material.

En el ámbito Internacional se conoce que Paulson y colaboradores de la Universidad de Wisconsin, Madison USA, han llevado a cabo investigaciones acerca de las ventajas de la caracterización zonal por FR y han logrado proponer un microscopio óptico de escaneo de campo cercano denominado NSOM (A nearfield scanning optical microscope), el cual utiliza el mecanismo de la Fotorreflectancia para obtener información acerca del campo eléctrico superficial a una temperatura constante para diferentes zonas del material semiconductor GaAs sin dopar (3). Sin embargo, este microscopio funciona para muestras en las cuales los espectros de FR exhiben una estructura correspondiente al régimen de campo medio, donde los espectros de FR exhiben oscilaciones tipo FranzKeldysh. Hasta donde conocemos no existen reportes de microscopios basados en la técnica de FR en el ámbito local e internacional, para el análisis de espectros en el régimen de campo bajo. De esta manera los resultados obtenidos mediante experimentos con él servomecanismo elaborado son muy importantes, porque abre la posibilidad de realizar trabajos de investigación, que permitan la obtención de un modelo físico y matemático para el análisis de imágenes de la variación zonal de la señal de FR en el régimen de campo bajo y una excelente oportunidad para publicar por primera vez imágenes de estudios zonales de la señal de FR en materiales semiconductores.

Por otro lado, este artículo se encuentra distribuido por títulos, de la siguiente manera: el segundo título "Conceptos", aborda aspectos conceptuales importantes en el desarrollo de este trabajo; como la técnica de Fotorreflectancia y la automatización. En el tercer título se encuentra la "Metodología", donde se hace una descripción del generador de trayectoria, el control digital y el servomecanismo requerido en el control de la posición de la muestra. El cuarto título presenta los "Resultados y discusión". En el quinto título, encontramos las "Conclusiones" obtenidas de la realización del presente trabajo. Finalmente, el lector encontrará el soporte bibliográfico.

\section{CONCEPTOS}

\section{A. Técnica de Fotorreffectancia}

La FR es una técnica de reflectividad modulada, en donde el parámetro de modulación es el campo eléctrico superficial. Dicha modulación se induce por medio de la interrupción periódica de un haz de luz láser, denominado haz de modulación. Tal como se muestra en la Figura 1, se emplea otro haz de luz monocromática proveniente de una lámpara QTH, llamada haz de 
prueba, el cual es reflejado por el material y recibido posteriormente por un detector. En el detector el haz de modulación debe eliminarse, de allí que se use un filtro longpass a la entrada del detector; por su parte, el haz de prueba reflejado en la muestra contiene información acerca del cambio en la reflectividad $\Delta R$ y la reflectividad $\mathrm{R}$ (4).

El mecanismo de la FR es la modulación del campo eléctrico superficial a través de la recombinación de portadores minoritarios en trampas de la superficie. Los pares electrón-hueco, generados cuando la

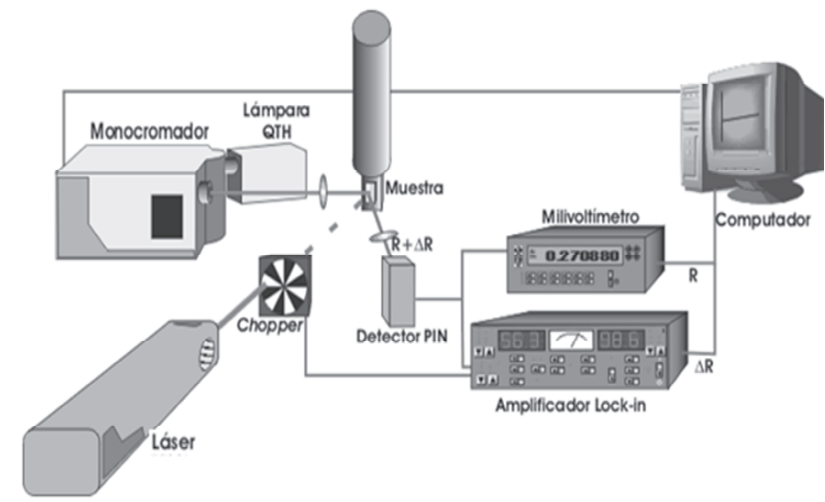

Figura 1. Diagrama en bloques del arreglo experimental de la técnica de Fotorreflectancia (5).

luz moduladora incide sobre la muestra, están en la posibilidad de ocupar trampas, modificando de esta manera el campo eléctrico superficial. En el régimen de campo bajo, la forma de línea del espectro de FR puede ser descrita por (6):

$$
\frac{\Delta R}{R}=R e\left[\sum_{J=1}^{P} C J \exp \left(i \theta^{j}\right)\left(E^{-} E_{p c j}+i \Gamma_{j}\right)^{-m j}\right]
$$

Donde $\mathrm{C}_{\mathrm{j}}$ es la amplitud, $\theta_{\mathrm{j}}$ el ángulo de fase, $\Gamma_{\mathrm{j}}$ parámetro fenomenológico de ensanchamiento, $\mathrm{E}_{\mathrm{g}}$ energía de la $j$-esima transición y $m$ el punto crítico (si $m=2.5$, transición interbanda, $m=2.0$, transición excitonica).

\section{B. Automatización Electrónica}

En el campo de la ingeniería electrónica es de gran importancia la automatización de procesos como por ejemplo: el posicionamiento robótico de mecanismos para tareas específicas, control numérico de las máquinas y herramienta de las industrias de manufactura. En el caso de este trabajo, para realizar la automatización electrónica de la caracterización zonal de semiconductores, se construyó un control para un servomecanismo. Los servomecanismos (diferente de servomotor) se pueden obtener de la combinación de piezas mecánicas y dispositivos electrónicos como motores, entre otros; y han sido ampliamente utilizados en robots de parte móvil o fija (7). Estos sistemas también pueden estar formados por partes neumáticas o hidráulicas y contar con un controlador de precisión.

\section{METODOLOGIA}

En la actualidad, el Instituto interdisciplinario de las Ciencias de la universidad del Quindío ha logrado implementar la técnica de FR, la cual se encuentra calibrada en los rangos espectrales del visible e infrarrojo cercano. En la técnica de FR implementada, los haces de luz son dirigidos a la muestra mediante espejos y lentes. La automatización electrónica realizada al montaje experimental de la técnica de FR, consistió en agregar al camino óptico de la técnica de FR un sistema electromecánico para el control digital del posicionamiento de la muestra, dejando fija la zona de incidencia de los haces de luz, lo cual permitió desplazar en un sistema coordenado X e Y, la muestra bajo estudio, como se presenta en la Figura 2 la trayectoria descrita, permitiendo así la caracterización zonal de materiales semiconductores de una manera automatizada.

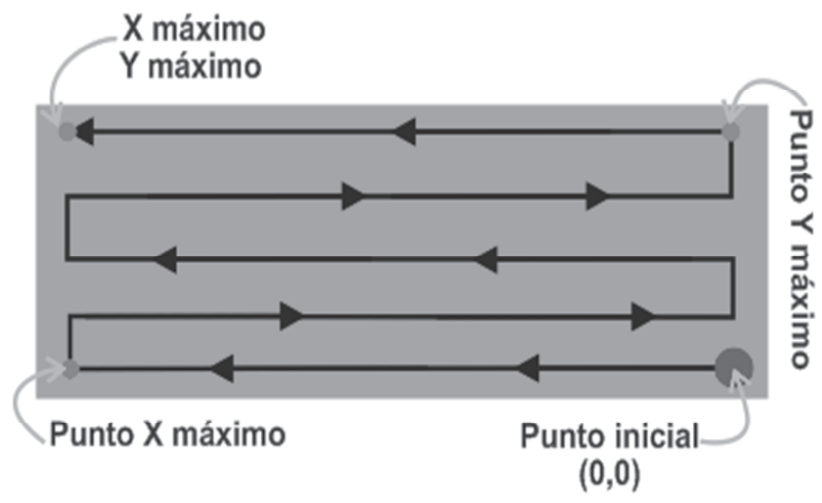

a)

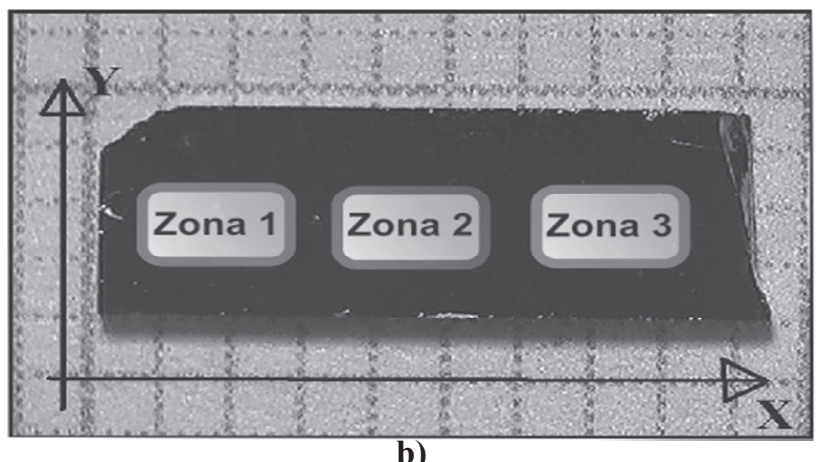

b)

Figura 2. Muestra de GaAs estudiada $(8,9)$ : a) Trayectoria del escaneo sobre la muestra semiconductora, b) Muestra semiconductora de GaAs y zonas estudiadas. 
Para realizar la automatización electrónica de la caracterización zonal de semiconductores, se construyó un control para un servomecanismo (motor + montaje mecánico) bajo la configuración básica de diagramas en bloque presentado en la Figura 3, que corresponde a un diagrama en bloques del control en lazo abierto (10) para un servomecanismo. Es bien conocido que un control de servomecanismo involucra en primer lugar un generador de trayectoria, en segundo lugar el control de posición que se realizó empleando un programa elaborado en el software LabVIEW (11), en tercer lugar el driver de potencia, y finalmente el motor paso a paso, el cual se acopla mecánicamente al montaje por medio de una línea de transmisión mecánica.

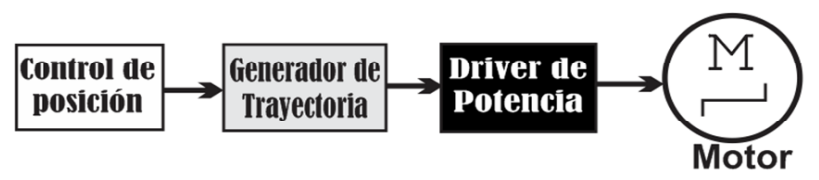

Figura 3. Diagrama en bloques del control básico de un servomecanismo.

En la Figura 4 a) se presenta el montaje mecánico por partes, donde se puede observar que la pieza 1 corresponde al posicionador micrométrico de la serie MT-XY de la empresa Newport, el cual fue acoplado directamente a motores paso a paso, de tal manera que se logró el desplazamiento del porta muestras en los ejes coordenados XY; la pieza 2 corresponde al motor paso a paso del eje Y; la 3 corresponde al motor paso a paso del eje X; la 4 corresponde al acople mecánico directo (12); la 5 corresponde a la línea de transmisión mecánica tipo brístol de 2,2 $\mathrm{mm}$; las piezas 6 y 7 corresponden a barras de acero empleadas como estructura del montaje mecánico, mientras que la pieza 8 corresponde a la base de aluminio empleada como porta muestras o platina de aluminio de $10 \mathrm{~cm}$ de largo por $5 \mathrm{~cm}$ de ancho.

En la Figura 4 , se puede observar el montaje experimental del servomecanismo construido en este trabajo.

Estas modificaciones al arreglo experimental de la Figura 1, permitieron obtener el montaje experimental de la Figura 5. En este montaje, la muestra es ubicada en un posicionador XY; además, se emplearon dos motores paso a paso para el movimiento de la muestra en los ejes XY, cuyas resoluciones fueron de $1.8^{\circ}$; aunque éstas se pueden modificar por software. Para el funcionamiento de los motores se desarrolló

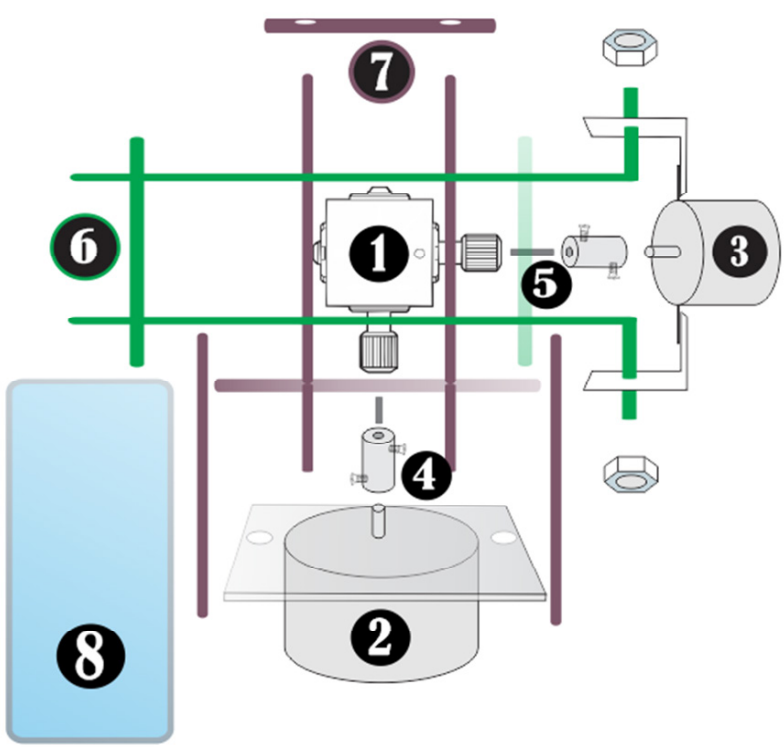

a)

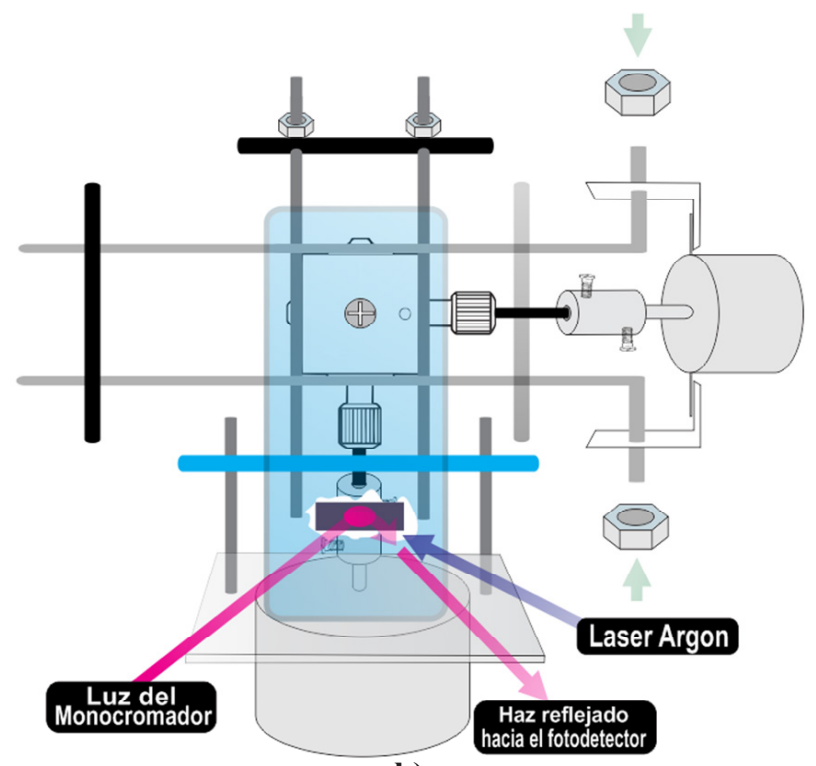

b)

Figura 4. Servomecanismo construido. a) Diagrama en bloques del servomecanismo b) Servomecanismo ensamblado.

e implementó un driver de potencia eléctrica. Las señales de control del generador de trayectoria y la comunicación con el PC (Personal Computer) fueron suministradas por un sistema electrónico embebido, basado en el microcontrolador AVR (13) ATmega 16 de la empresa ATMEL. El sistema electrónico obtenido permitió la generación de la trayectoria del porta muestras para lo cual se realizó una herramienta de visualización elaborada en el software LabVIEW que permitió el control de la posición en los ejes XY. 


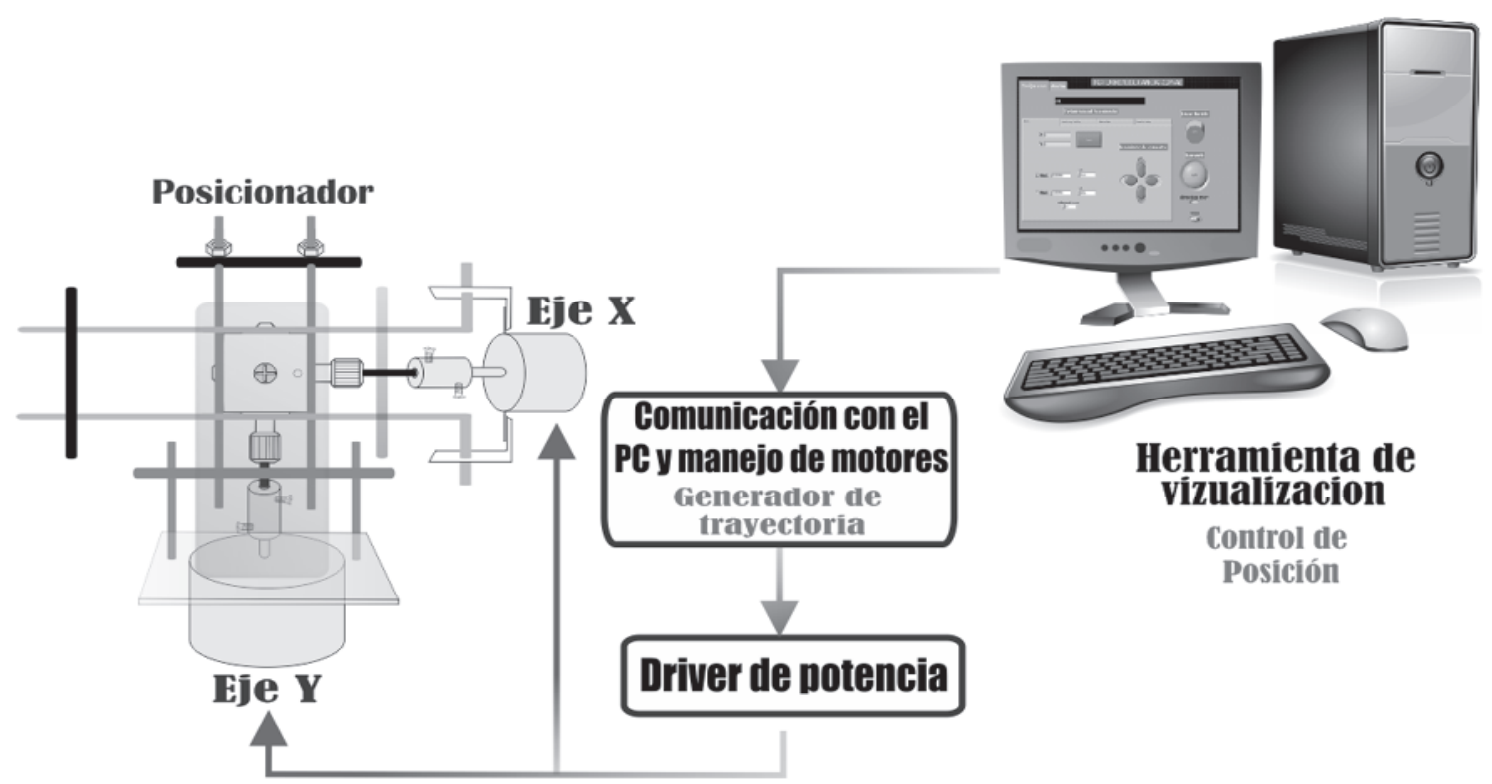

Figura 5. Diagrama en bloques del arreglo experimental obtenido mediante la realización de este trabajo. (14).

En la Figura 6 se presenta el diagrama de flujo correspondiente al generador de trayectoria donde se muestra la configuración de los puertos usados como salidas y entradas, respectivamente. Además, se empleó un método indirecto para medir la posición de la muestra en los ejes XY que consistió en contabilizar los pasos de los motores para una distancia fija de 9 mm y se utilizó un análisis estadístico del comportamiento aleatorio de la información recopilada, encontrándose que el sistema electromecánico registró una resolución alrededor de $1.0 \mu \mathrm{m}$ con un error inferior a $\pm 0.1 \mu \mathrm{m}$, como se ilustra en la Figura 7 .

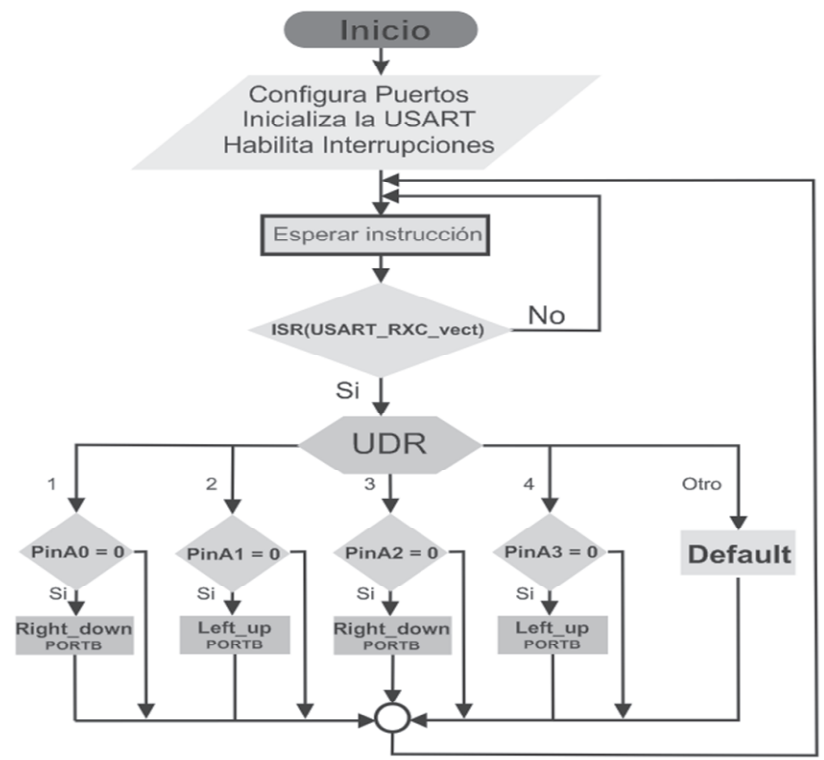

Figura 6. Diagrama de flujo para el protocolo generador de trayectoria.

\section{RESULTADOS Y DISCUSIÓN}

En la Figura 8 se presenta el sistema electromecánico construido en este trabajo. La muestra fue ubicada en el porta muestras empleando silicona de alta conductividad térmica; además, se puede observar la disposición del posicionador mecánico y sus acoples mecánicos, así como los finales de carrera que permi-

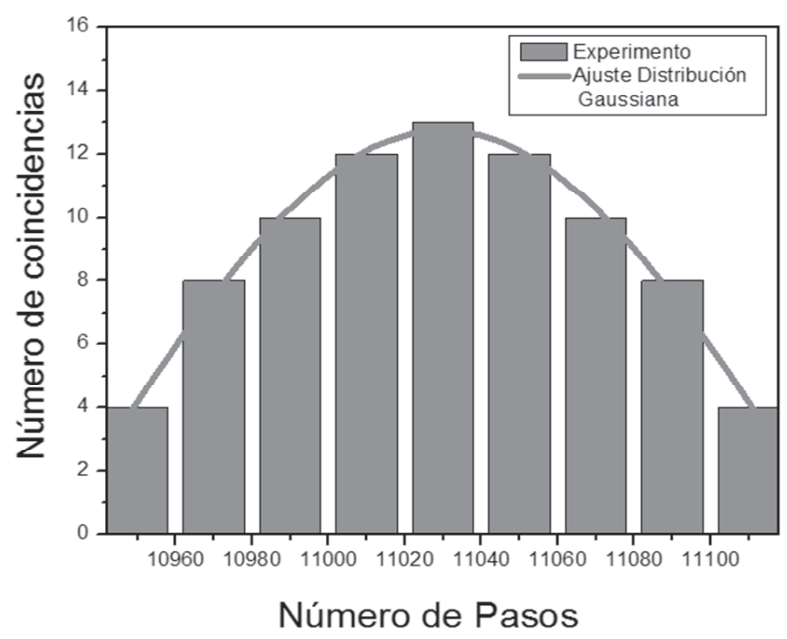

Figura 7. Comportamiento aleatorio del número de paso en el sistema electromecánico propuesto en este trabajo.

ten un desplazamiento lineal hasta de $9 \mathrm{~mm}$ por eje. Para energizar los motores paso a paso y la tarjeta FRZ_2010 se construyó una fuente con salida dual regulada a $+12 \mathrm{~V}$ y $-12 \mathrm{~V}$ y dos salidas de voltajes fijos a $5 \mathrm{~V}$ y $12 \mathrm{~V}$; mientras que en la Figura 9 se visualiza la tarjeta de circuito impreso denominada FRZ1_2010 
Una vez puesto en funcionamiento el sistema diseñado, se estableció un procedimiento para tomar las medidas de caracterización zonal de materiales semiconductores, siguiendo los protocolos de la técnica de Fotorreflectancia implementada en el Instituto Interdisciplinario de las Ciencias.

En la Figura 10 se presentan los espectros de FR obtenidas en un monocristal comercial de GaAs a 300 $\mathrm{K}$, para tres zonas diferentes.

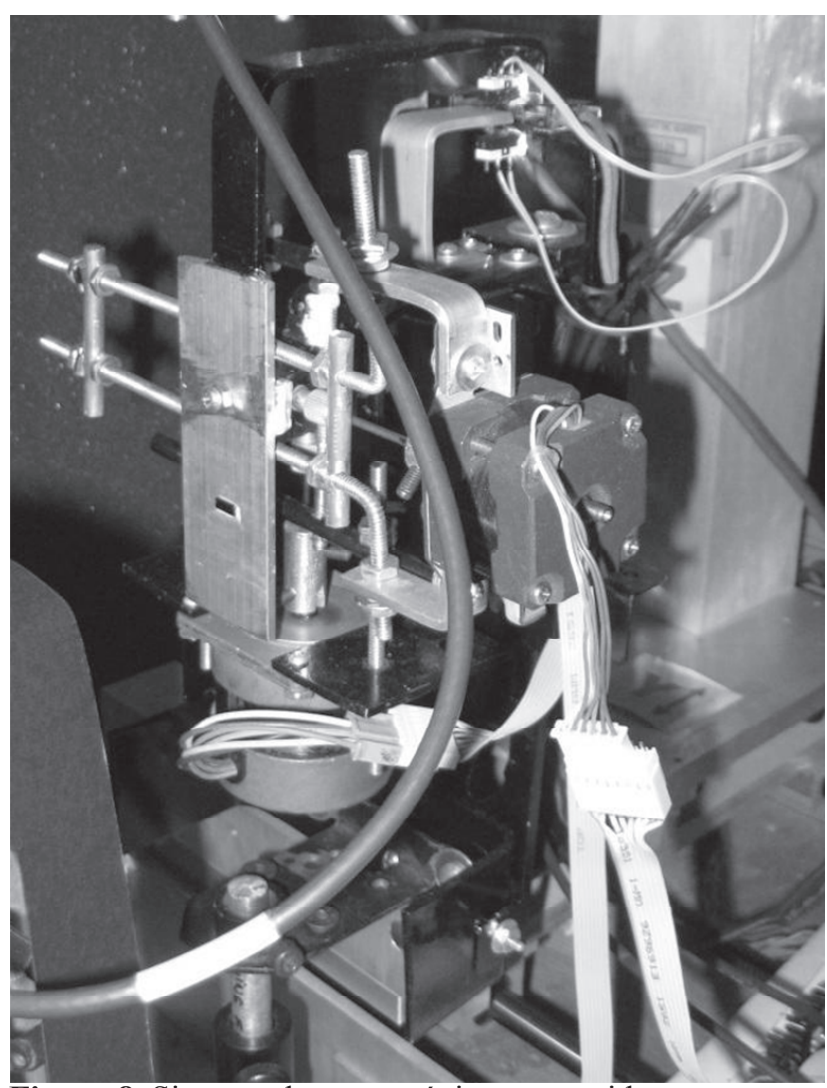

Figura 8. Sistema electromecánico construido en este proyecto.

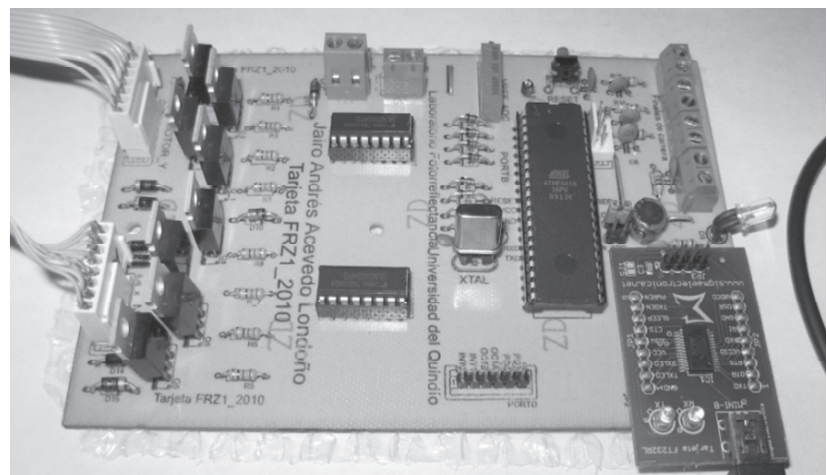

Figura 9. Tarjeta construida denominada FRZ1_2010.

Se puede observar en estos espectros que la energía del gap se encuentra alrededor de $1,41 \mathrm{eV}$, el cual corresponde al valor de la energía de transición interbanda, reportada para este material $(7,8)$.
Es importante señalar que los espectros de FR mantienen su estructura, no presentan corrimientos en energía y lo único que cambian son sus amplitudes, que pueden ser atribuidas a la presencia de portadores libres y su variación zonal, en el régimen de campo bajo.

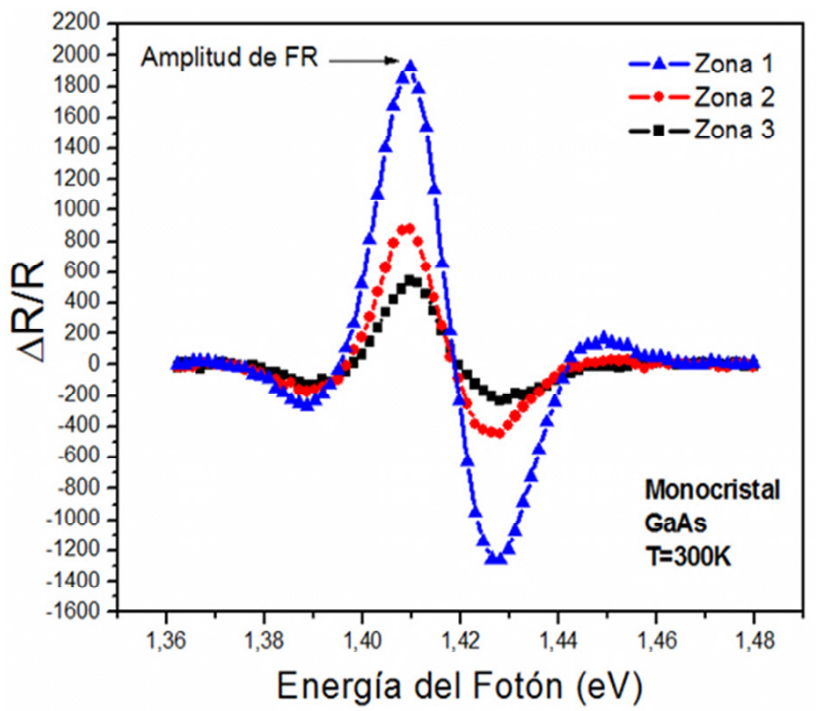

Figura 10. Espectros de FR de la muestra de GaAs para las tres zonas estudiadas

Por último, en las Figuras 11 a) y b) se presentan imágenes de la variación zonal de la señal de FR en la muestra comercial de GaAs sin rotar y rotada a $90^{\circ}$, respectivamente. En estas imágenes se han indicado las diferentes zonas estudiadas donde se puede apreciar el efecto de la variación zonal del máximo de la amplitud de la señal de FR.

Así mismo, siguiendo este mismo procedimiento, en las Figuras 11 c) y d) se presentan las variaciones zonales de la señal de FR, para imágenes en 2D obtenidas empleando el software Matlab 7.6 de la empresa the Mathworks. Se puede observar como los colores permiten diferenciar las áreas de mayor amplitud de la señal de FR (14).

De nuevo la zona 1 sigue exhibiendo la mayor señal de FR. Este resultado es muy importante, porque abre la posibilidad de generar otros trabajos relacionados con el procesamiento de este tipo de imágenes.

Por consiguiente se ha encontrado que la zona 1 presenta mayor amplitud en la señal de FR que las otras zonas asociándose, de manera cualitativa, a una mayor densidad de portadores libres. La obtención de un modelo físico es una propuesta abierta para el análisis 
de imágenes de la variación zonal de la señal de FR en el régimen de campo bajo, tal como ha sido propuesto recientemente, para el régimen de campo medio por O. S. KomKov et al (15).

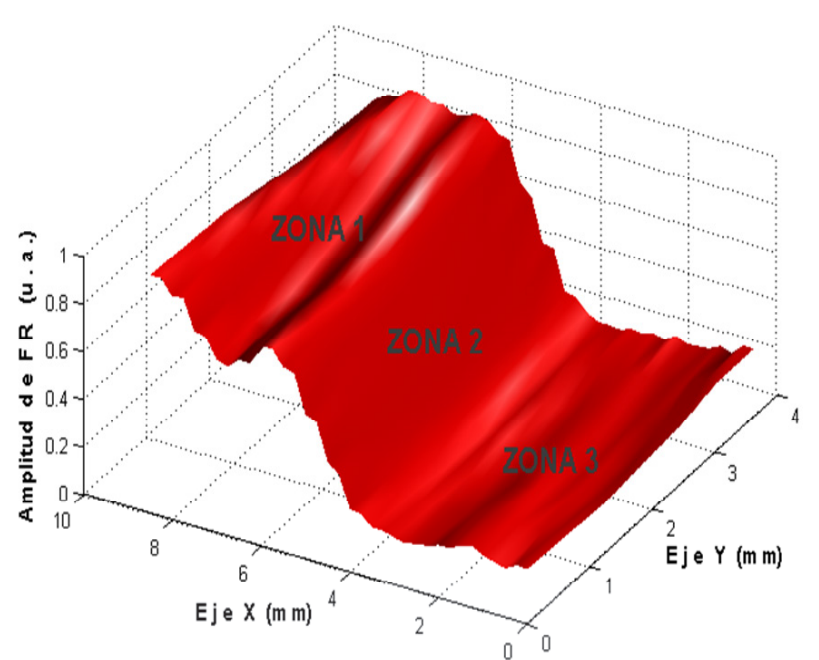

a)

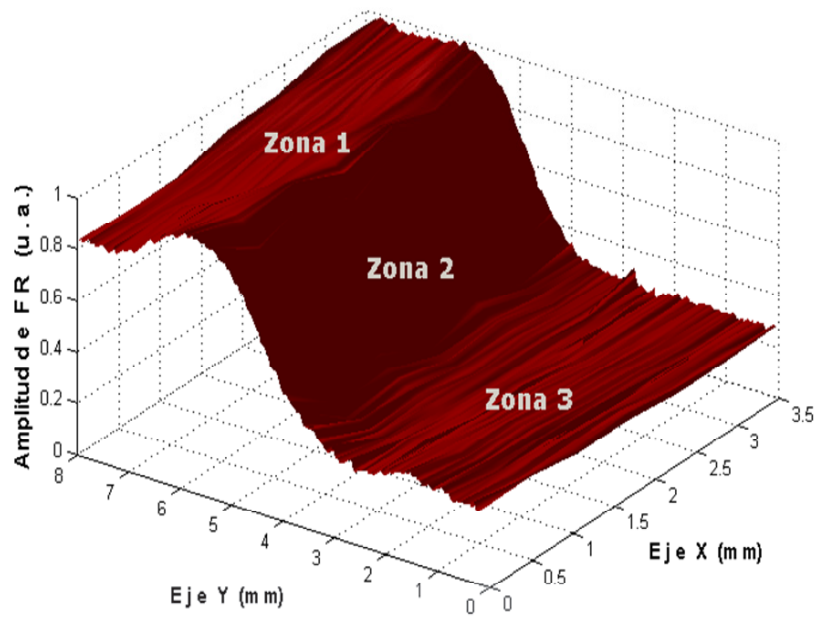

b)

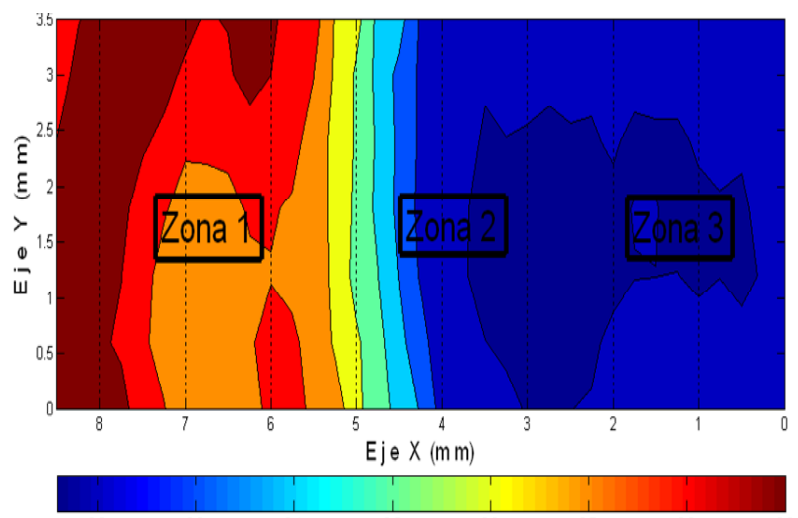

c)

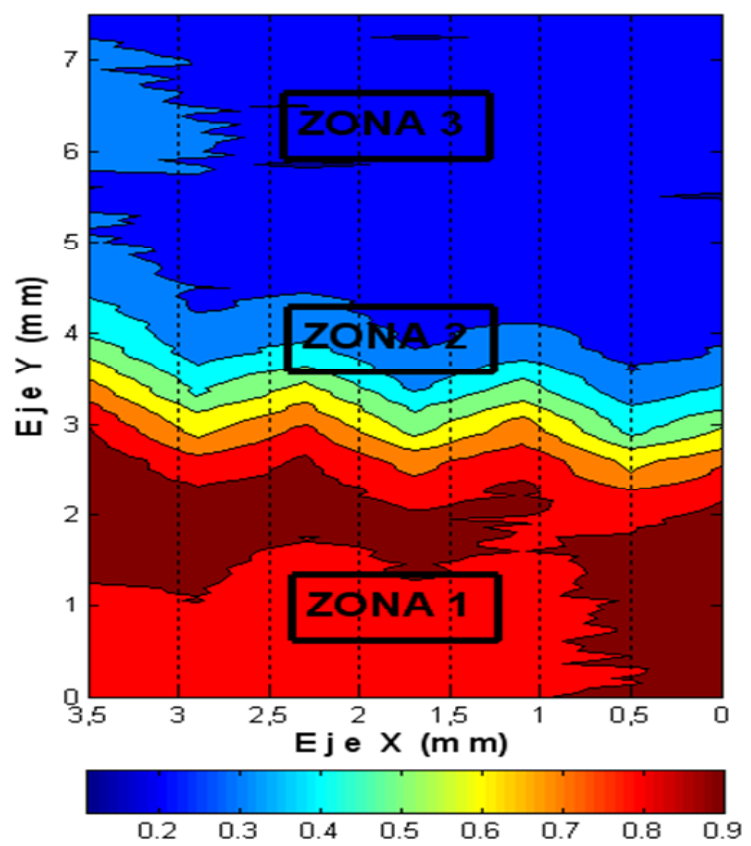

d)

Figura 11. Variación zonal en la muestra estudiada. a) Variación zonal de la amplitud de FR en una muestra de GaAs para las zonas estudiadas. b) Variación zonal de la amplitud de FR en una muestra de GaAs al rotar $90^{\circ}$ la posición de la muestra en el porta muestras. c) Imagen en 2D de la caracterización zonal por FR en el régimen de campo bajo, para una muestra de GaAs en posición horizontal. d) Imagen en 2D de la caracterización zonal por FR en el régimen de campo bajo, para una muestra de GaAs en posición vertical.

\section{CONCLUSIONES}

Se realizó la automatización electrónica de la técnica de FR para la caracterización zonal de materiales semiconductores, mediante el diseño y elaboración de un servomecanismo controlado por una interface digital, la cual permitió obtener la variación zonal de la amplitud de la señal de FR en la superficie de una muestra de GaAs comercial, lográndose identificar una zona de mayor amplitud de la señal de FR, la cual se asoció de manera cualitativa a una zona de mayor densidad de portadores libres en la superficie de la muestra.

Se implementó un control digital de la posición de un porta muestras basado en una alternativa de generador de trayectoria, lográndose el uso de tecnología ATMEGA 16 y un puerto de comunicaciones por conector USB. 
Se elaboró una herramienta de visualización en el software LabVIEW que permitió obtener, de una manera automatizada, imágenes de la caracterización zonal por FR del material semiconductor GaAs que concuerda con la reportada en la literatura especializada. Se encontró que la variación zonal de la amplitud de
FR es independiente de la rotación de la muestra y que el sistema electromecánico propuesto presentó una resolución espacial de $1.0 \mu \mathrm{m}$ en ambos ejes. De esta manera se ha contribuido al incremento de la versatilidad de la técnica de FR en el Instituto Interdisciplinario de las Ciencias.

\section{BIBLIOGRAFIA}

1. Pollak FH, Shen H. Modulation spectroscopy of semiconductors: bulk/thin film, microstructures, surfaces/interfaces and devices. Mater. Sci. Eng. 1993; 10(7-8):15.

2. Misiewicz J., Sitarek P., Sek G., Kudrawiec R. Semiconductor heterostructures and device structures investigated by photoreflectance spectroscopy. Mater Sci. 2003; 21(3): 263.

3. Paulson C, Ellis AB, McCaughan L, Hawkins B, Sun J, Kuech TF. Demonstration of nearfield scanning photoreflectance spectroscopy. Appl Phys. 2000; 77: 1943.

4. F. H. Pollak and H. Shen. Mater. Sci. Eng. 1993; 10: 275.

5. Torres L, Prías-Barragán JJ, Espinosa-Arbeláez DG, Alvares GA, Ariza-Calderón H. Adaptación del software LabVIEW para la automatización de la técnica de Fotorreflectancia. Revista de Divulgación científica y Tecnológica instituto interdisciplinario de las ciencias. 2008; 5(10)

6. Aspnes FH, Studna A. Phys. Rev. B. 1963; 7: 4605

7. Mcmillan GK, Considine DM. Process / Industrial instruments and controls handbook. 5a ed. Estados Unidos: McGraw-Hill; 1999. p 9.82-9.102.

8. Aspnes DE, Studna AA. Schottky-barrier electroreflectance: application to GaAs. Phys. Rev. B. 1973; 7(10): 4605.

9. Adachi S. Band gaps and refractive indices of GaAlAsSb, GISA and InPAsSb: key properties for a variety of the 2-4 $\mu \mathrm{m}$ optoelectronic device applications. J Appl Phys. 1987; 61(10): 4869.

10. Ogata K. Ingeniería de Control moderna. 3a ed. Méjico: Editorial Prentice Hall; 1995.

11. J. A. Acevedo-Londoño, J. J. Prías-Barragán. Elaboración de una Herramienta de visualización en Labview para la caracterización zonal de materiales semiconductores por la técnica de fotorreflectancia. Revista de Divulgación científica y Tecnológica instituto interdisciplinario de las ciencias. 2009; 6(1): 41-52.

12. Quilodra V, Moreno PR, Marchan LC. Acoplamientos mecánicos. Chile: Universidad Tecnológica de Chile; 2005.

13. Mazidi AM, Mazidi J, Naimi S, Naimi S. Avr Microcontroller and Embedded Systems: Using Assembly and C. Estados Unidos: Prentice Hall; 2011.

14. Acevedo-Londoño JA, Prías-Barragán JJ, Torres-Londoño L, Ariza-Calderón H. Automatización electrónica de la técnica de fotorreflectancia para la caracterización zonal de materiales semiconductores. Rev Col Fis. 2009; 41 (2): 3-4.

15. Komkov OS, Pikhtin AN, Zhilyaev YuV, Fedorov LM. Determination of the Free Carrier Concentration in Ultra-Pure GaAs Epilayers by a Photoreflectance Technique. Tech Phys Lett. 2008; 34 (1): 37-39. 